\title{
An Enhanced Online Learning Environment in the Time of COVID- 19
}

\author{
Shimaa Ouf \\ Faculty of Commerce and Business Administration \\ Business Information Systems Department, Helwan University, Egypt \\ E-mail: shimaaouf@yahoo.com
}

Keywords: Covid-19, learning process, blockchain, semantic web, digital certificate, ontologies

Received: October 6, 2020

\begin{abstract}
The coronavirus has forced educational institutions and universities all around the world to close their doors. There are about 1.2 billion learners outside of educational institutions in 191 nations around the world. To ensure the safety of workers, learners, and educators, the world is currently converting training, learning, and work online to reduce total disease mortality and flatten the infection curve. This paper examines the problems of the existing learning environment and proposes a framework to address them to make the transition to online learning simple, manageable, secure, and effective. Securing private data, forged certifications, payment systems, tracking student behavior, low quality of the learning process, and centralization of authority are all issues that learning environments confront. Educational institutions must build efficient, effective, transparent, immutable, and secure learning environments. We give a comprehensive analysis that introduces research papers that address learning-related concerns. Most of this research has focused on leveraging blockchain technology to improve the learning process while ignoring other crucial technologies such as the semantic web. The semantic web and blockchain will be combined to form a semantic blockchain. The semantic web is being utilized to improve the representation of learning processes based on blockchain, such as annotations, and to enable reasoning, inferences, and queries using SPARQL. In the event of a COVID-19 pandemic, the semantic blockchain will aid educational institutions in improving their learning processes. It is seen as a critical component of the next generation of e-learning systems. The proposed framework for improving the learning process via a semantic blockchain is presented in this paper

Povzetek: V času kovida je pogosto učenje na daljavo in prispevek skuša opisati varno okolje s pomočjo semantičnih verižnih tehnologij.
\end{abstract}

\section{Introduction}

The World Health Organization has declared a worldwide flare-up of covid-19; as a result, people across the world are confronting a huge issue. Coronavirus harms our lifestyles and proclivities, as well as our ability to adapt and be resilient. The countries most affected by the coronavirus pandemic are experiencing a severe public health crisis, which will have a long-term impact on their economic and social structures. Respect for the appropriation of social distancing and lockdown is required by the new regulations to combat this sickness and limit its spread. This changes the way learning objects, learning activities, and teaching methods are fulfilled and delivered [1-2].

In the time of COVID-19 and to slow and limit the spread of the Coronavirus, online learning becomes a necessity; it is no more an option. Such online learning is required in this deadly virus where video conferencing is possible with learners, discussions with learners can be available, lectures can be accessed on mobile phones as well and not just laptops, the ability to view lectures already recorded. Hence, this paper focuses on analysing the challenges within the online learning environment and gives an arrangement to these challenges.

The existing learning environment faces numerous issues [3], including trouble verifying certifications due to the addition of unearned qualifications and the theft of student data, and the centralized learning process. In addition, LinkedIn's online learning site Lynda was hacked in December 2016. The data of the students was obtained by an unauthorized third party. As a result of the centralized storage of the Lynda platform, which gives single attack points and access to all stored data, the 9.5 million learner accounts are affected. Recently, the University of California-Berkeley, WisconsinMilwaukee, Ohio State University, and University of Kirkwood Community College have been hacked. The University of Indiana kept crucial information on an open website. Data was saved on tapes or computers at wellknown universities such as Stanford University, Miami University, and Utah Hospitals and Clinics, which were later hacked [4]. There is a need to improve data interoperability by increasing the representation capabilities of learning process-based blockchain 
knowledge that is obtained from many sources by annotating its concepts, sub-concepts, and relationships with semantically rich languages. Combining blockchain technology with learning environments is thought to have a high level of security. Data protection, privacy, integrity, and creating trust between all parties involved in the network are all aspects of security. Blockchain is based on a peer-to-peer network in which diverse participants, assets, and transactions are linked. It allows for more control over how and by whom learners' data is accessible [5].

Semantic web standards are employed in this paper to introduce common data formats for a learning processbased blockchain that allows data to be shared and reused [6].

In a summary, one of the major design ideas of the Semantic Web is that data can be published anywhere on the internet by anybody and that it should be easy to query and incorporate that data without having to aggregate it all in one location. Semantic blockchain introduces the decentralized, transparent, and available architecture of the learning process. Decentralization means there are no central attack points, and each node has an independent copy of the blockchain and verifies it against all others. Transparency means that each participant can track the transaction data recorded in the blockchain network and check the data changes. Availability means that a distributed system is stored on different devices and continues to run even if some systems become unavailable [7-9]. In this paper, semantic blockchain facilitates tracing and tracking all the activities in the learning process which include learners' achievements due to authenticating learners' identities, their payment data, online exams, digital certificates, digital courses, open badges, learning activities, and teaching methods. The legalized educational institution and the learners are permitted to access and edit the stored data in the semantic blockchain under some restricted rules and conditions as shown in figure 1 .

The research community shows that online learning has been existed to increase information retention, and save time, meaning the changes covid-19 has caused might be here to stay.

\section{Literature review}

The current research studies that preview the essential role that blockchain plays in the learning environment are presented in this part.

The authors of this paper reveal that China and Italy were among the first countries to be hit by the COVID-19 pandemic. It focuses on learners' and educators' needs to modify their lifestyles and routines, and it pushes them toward online learning [1]. It covers the difficulties that exist in today's online learning environment. The authors of this article used the blockchain to create and democratise educational reputation.

The blockchain technology is used to hold secure and immutable educational achievement records, such as degrees, certificates, and educational attainment, that are accessible and distributed across several institutions. The digital certificate is also added to the blockchain by the awarding institution. The certificate can be accessed via a link from an online CV or shared with employers [9]. Authors in this paper integrate the Internet of Things (IoT) with blockchain to create smart and trustworthy environments. In an intelligent educational system, IoT facilitates data exchange by using connected objects and sensors through smart devices [10].

In this paper and based on the European Credit Transfer and Accumulation System (ECTS), the authors

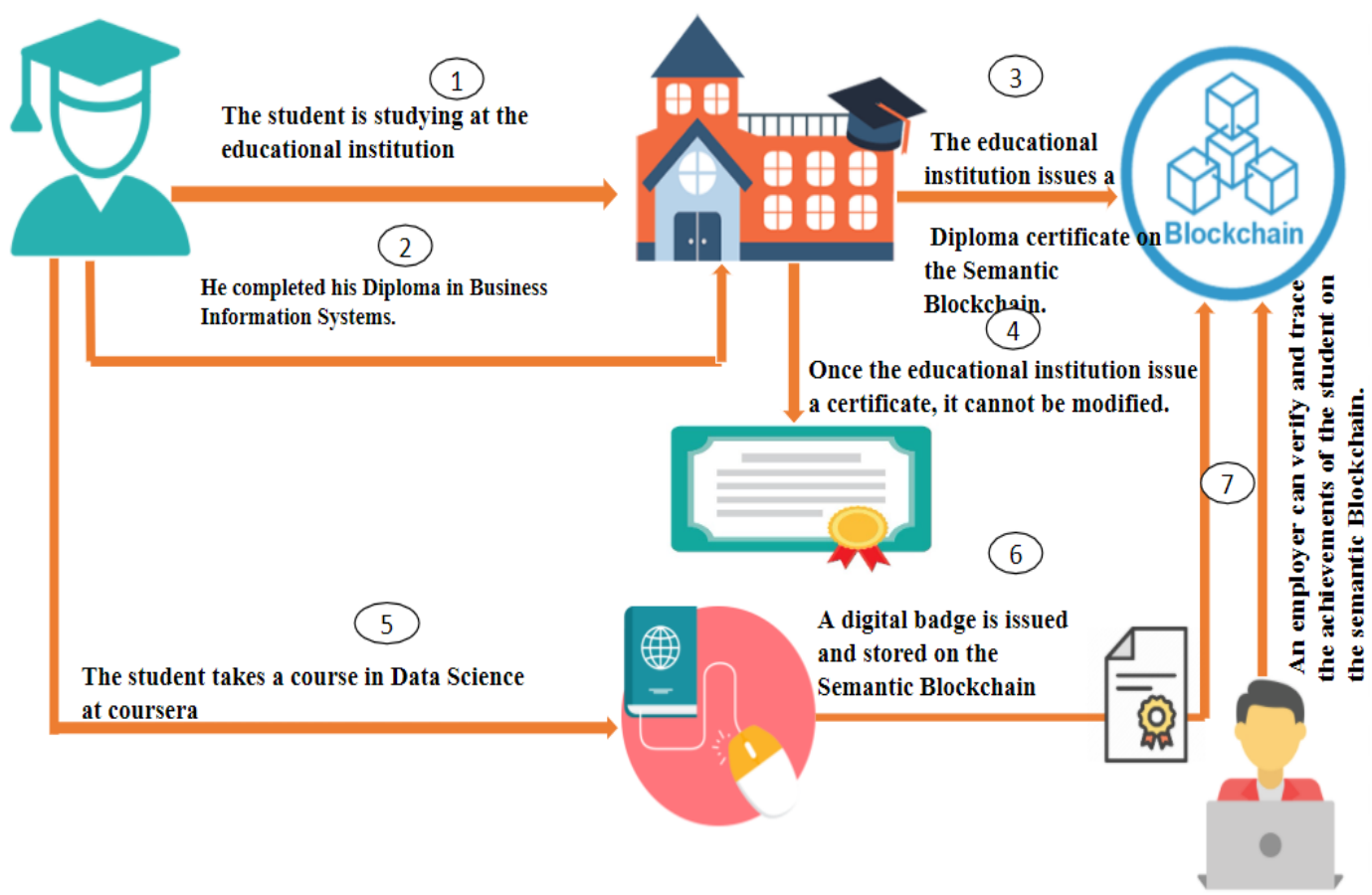

Figure 1: An excerpt of student transactions within semantic blockchain. 
utilize blockchain technology to show a worldwide stage of higher education credit, named EduCTX. The proposed stage presented a universally permanent, trusted, straightforward decentralized credit and grading system that can present a globally unified viewpoint for learners and higher education institutions, as well as for other potential participants such as companies, institutions, and organizations [12].

Blockchain is being used to improve the performance of virtual learning environments. This paper introduces the challenges of the current competition models. These models have the issues of untrustworthy information, unscientific competition, and unauthorized assessment that make learners and teachers much less enthusiastic. Blockchain is used to increase competition, assessment to enhance performance, transparency, and credibility. Besides, in this paper blockchain is used to avoid the problem of opaque and falsification messages, provide unchangeable digital certification of academic achievement [13]. Blockchain technology is considered one of the most revolutionary emerging hotspot technologies in different sectors. Due to its important features like distribution, decentralization, and tamperresistant features. Authors in this paper integrate IoT and blockchain technologies to introduce a reliable and secure model to eliminate the need for the third part while providing trusted and secured transactions. The proposed system is applied to an environment containing several connected sensors and uses blockchain technology, to develop a secure and distributed learning environment that checks the provenance data from the connected objects and ensures secure educational services [14].

Because of issues with digital rights management systems in an online learning environment, such as digital copyrights, infringement of multimedia courses, and counterfeit digital certificates. The authors of this study present a digital rights management system based on blockchain for securing content in an online learning environment via digital certificates validation [15].

The authors look at research publications that use blockchain technology in a classroom setting. They divide these publications into two categories. 1) An approach that is focused on the institution, and 2) an approach that is focused on the learner. Learner-centric blockchain enables the certification of a learner's credentials without the need for an intermediary (university) and secures digital certificates. Institution-centric solutions primarily manage university-related tasks such as payment processing and the storage of immutable student achievement data. The papers reviewed demonstrate the benefits of implementing blockchain technology in an educational setting to provide transparency, immutability, and trust [16].

The authors of this research looked at difficulties that educational institutions face, such as the possibility of manipulation and the difficulty of verifying and transmitting educational data between institutions. They propose a thorough literature study that highlights the importance of incorporating blockchain's benefits such as decentralization, traceability, and consensus mechanism into educational institutions' challenges. The blockchain technology allows for the decentralized and interoperable maintenance of learners' records [17].

Universities have recently received funding to establish campuses in many cities and nations. As a result, safeguarding online learning transactions such as learner profiles and certifications is becoming a top priority for security professionals. As a result, the purpose of this study is to highlight studies that use blockchain in a higher-inculcation context. The findings of these research demonstrate the advantages of implementing blockchain technology in higher education. The transaction data pertaining to certificates and money transfers, learner profiles and records, digital badges, publications, and human resources is secured by blockchain [18].

Authors in this paper mentioned that the blockchain consensus algorithm is used to bring many benefits such as consistent data with reduced errors, which solve trust and security problems by tracking sensor data measurements. They propose an intelligent educational system that can transfer and exchange data securely via a blockchain without the need for a third party to guarantee trust. A smart contract is used to ensure data integrity in peer-to-peer networks. As a result, the deployment costs of all components of the intelligent educational system can be reduced. The system is evaluated based on a set of criteria, such as integrity, confidentiality, and availability to confirm that the entire transaction is done without any alteration. Confidentiality enables the authorized peers to access the messages and finally, the availability guarantees that the services and data are available anywhere anytime. This evaluation reflects that our blockchain-based decentralized intelligent system introduces a marginal increase in transaction processing [19].

The authors propose a blockchain-based framework consists of three layers, which are the data layer, logic layer, and application layer. These layers represent the educational process and the evaluation process of smart education. Blockchain is used for storing achievements, credits, certificates, and grades and improve the safety and security of the education system [20].

The authors implement an Education-Industry cooperative system predicated on blockchain technology. Hyperledger framework is utilized to offer transparency and non-tampering features to the Certificate Ascendancy accommodation and transactions. It simulates the roles of companies and universities in the system and enables them to apportion information transparently, which achieves information symmetry among learner adeptness and cognizance information, the ordinant dictations of a company's recruitment, and current market trends [21].

The authors propose an educational framework predicated on a globally trusted blockchain. The global network includes many participants like universities, companies, and other higher scholastic institutions. It will work in a homogeneous environment where all participants collaborate and interact. The proposed framework enables an ecumenical view of a learner's performance. It secures the cognition objects and issues digital certificates for a registered learner which can be digitally transferred among other organizations [22]. 


\begin{tabular}{|c|c|c|c|c|c|c|c|c|}
\hline Author/Learning Process Components & & 递苞 & 象: & 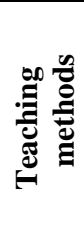 & 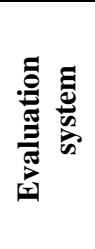 & 莺 & 牙 & 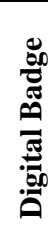 \\
\hline Sharples and Domingue 2016 & $\sqrt{ }$ & $\times$ & $\times$ & $\times$ & $x$ & $\times$ & $\sqrt{ }$ & $\sqrt{ }$ \\
\hline Bdiwi, De Runz et al. 2017 & $\sqrt{ }$ & $\times$ & $\times$ & $x$ & $x$ & $\times$ & $x$ & $\times$ \\
\hline Duan, Zhong et al. 2017 & $\sqrt{ }$ & $\sqrt{ }$ & $x$ & $x$ & $x$ & $x$ & $x$ & $\times$ \\
\hline Turkanović, Hölbl et al. 2018 & $\sqrt{ }$ & $x$ & $x$ & $x$ & $x$ & $\sqrt{ }$ & $\sqrt{ }$ & $x$ \\
\hline $\mathrm{Wu}$ and $\mathrm{Li} 2018$ & $\sqrt{ }$ & $x$ & $x$ & $x$ & $\sqrt{ }$ & $x$ & $\sqrt{ }$ & $\times$ \\
\hline (Bdiwi, De Runz et al. 2018) & $\sqrt{ }$ & $x$ & $x$ & $x$ & $x$ & $x$ & $x$ & $\times$ \\
\hline Gong, Liu et al & $\sqrt{ }$ & $\sqrt{ }$ & $x$ & $x$ & $\sqrt{ }$ & $x$ & $\sqrt{ }$ & $x$ \\
\hline Liu, Guan et al. 2018 & $\sqrt{ }$ & $x$ & $x$ & $x$ & $x$ & $x$ & $\sqrt{ }$ & $x$ \\
\hline Srivastava, Bhattacharya et al. 2018 & $\sqrt{ }$ & $\sqrt{ }$ & $x$ & $x$ & $x$ & $\sqrt{ }$ & $\sqrt{ }$ & $x$ \\
\hline Mikroyannidis, Domingue et al. 2018 & $\sqrt{ }$ & $x$ & $x$ & $x$ & $x$ & $x$ & $x$ & $\sqrt{ }$ \\
\hline Mikroyannidis, Domingue et al. 2018 & $\sqrt{ }$ & $\sqrt{ }$ & $x$ & $x$ & $x$ & $x$ & $x$ & $\sqrt{ }$ \\
\hline Juričić, Radošević et al. 2019 & $\sqrt{ }$ & $\sqrt{ }$ & $x$ & $x$ & $x$ & $x$ & $\sqrt{ }$ & $\times$ \\
\hline Bdiwi, de Runz et al. 2019 & $\sqrt{ }$ & $x$ & $x$ & $x$ & $x$ & $x$ & $x$ & $x$ \\
\hline Guo, Li et al. 2019 & $\sqrt{ }$ & $\sqrt{ }$ & $x$ & $x$ & $x$ & $x$ & $\sqrt{ }$ & $\times$ \\
\hline Al Harthy, Al Shuhaimi et al. 2019 & $\sqrt{ }$ & $x$ & $x$ & $x$ & $x$ & $x$ & $\sqrt{ }$ & $x$ \\
\hline Our Proposed Framework & $\sqrt{ }$ & $\sqrt{ }$ & $\sqrt{ }$ & $\sqrt{ }$ & $\sqrt{ }$ & $\sqrt{ }$ & $\sqrt{ }$ & $\sqrt{ }$ \\
\hline
\end{tabular}

Table 1: The components of the learning process.

Open badges are a way to store formal and informal learning accreditation and assessment to provide a verifiable digital recognition of learning. Blockchain is utilized to secure immutable records of open badges. In this paper, the authors apply blockchain. It can fortify learners who want to advance their vocations in data science, by introducing them to recommended learning materials predicated on their achievements. Blockchain ameliorates data science accreditation and enables learners to benefit from an open and transparent accreditation system, as well as their vocations progress and receiving job recommendations that match their achievements. The authors use blockchain technology to develop a new environment of lifelong learning. Blockchain technology is used to manage learning objects; the way learners register and pay for them, as well as the accredited for their learning achievements. A new environment enables the learners to control their associated data at places them at the center of the learning process [23].

Higher education faces many challenges like no automated and centralization which means full control over the learners' knowledge. Blockchain plays an important role to solve these challenges. In this paper, the authors use blockchain to develop a decentralized education system that has many advantages like immutability, anonymity, integrity, credibility, and independence. There is no central authority and learners have complete control over their data. The proposed framework-based- blockchain has immutable records of official documents and certificates with complete confidence [24].

Table 1 shows the components of the learning process that were introduced in the literature review studies. In addition, we listed the components that authors discussed in their publications. We noted that none of the articles used blockchain technology to represent all aspects of the learning process (learner profile, learning objects, learning activities, teaching methods, evaluation system, payment system, digital certificate, and digital badge). They concentrated solely on one or more of the components. All aspects of the learning process will be represented using semantic blockchain in this paper.

Table 2 summarizes the technologies used by researchers to improve the learning process. We noticed that some studies used blockchain technology, others integrate IoT with blockchain, but all the studies ignored integrating blockchain with semantic web technology to enhance the quality of the learning process and make educational institutions achieve competitive advantages.

\section{Methodology}

This paper was performed based on searching for important articles. Electronic databases are a critical source of scientific research. The most comprehensive electronic databases, such as ScienceDirect, IEEE, and Springer, were searched to find relevant publications for our paper. The three databases are known for indexing high quality and high impact articles within the computer science field. The following keywords were used in the search: "Covid-19 AND Online Learning", "Blockchain AND Education", "Blockchain AND e-learning system", "Blockchain AND online learning", "Blockchain AND learning environment". The articles, which were related to our paper, are getting to be examined. All the retrieved articles were surveyed whereby unimportant papers and papers are not in English were excluded followed by 


\begin{tabular}{|c|c|c|c|}
\hline Author/Technology & IoT & Blockchian & Semantic Web \\
\hline (Sharples and Domingue 2016) & $\times$ & $\sqrt{ }$ & $\times$ \\
\hline (Bdiwi, De Runz et al. 2017) & $\sqrt{ }$ & $\times$ \\
\hline (Duan, Zhong et al. 2017) & $\times$ & $\sqrt{ }$ & $\times$ \\
\hline (Turkanović, Hölbl et al. 2018) & $\times$ & $\sqrt{ }$ & $\times$ \\
\hline (Wu and Li 2018) & $\times$ & $\sqrt{ }$ & $\times$ \\
\hline (Bdiwi, De Runz et al. 2018) & $\sqrt{ }$ & $\sqrt{ }$ & $\times$ \\
\hline (Gong, Liu et al.) & $\times$ & $\sqrt{ }$ & $\times$ \\
\hline (Liu, Guan et al. 2018) & $\times$ & $\sqrt{ }$ & $\times$ \\
\hline (Mrivastava, Bhattacharya et al. 2018) & $\times$ & $\sqrt{ }$ & $\times$ \\
\hline (Mikroyannidis, Domingue et al. 2018) & $\times$ & $\sqrt{ }$ & $\times$ \\
\hline (Juričić, Radošević et al. 2019) & $\times$ & $\sqrt{ }$ & $\times$ \\
\hline (Bdiwi, de Runz et al. 2019) & $\times$ & $\sqrt{ }$ & $\times$ \\
\hline (Guo, Li et al. 2019) & $\times$ & $\sqrt{ }$ & $\sqrt{ }$ \\
\hline (Al Harthy, Al Shuhaimi et al. 2019) & $\times$ & $\sqrt{ }$ & $\times$ \\
\hline Our Proposed Framework & $\sqrt{ }$ & $\times$ & \\
\hline
\end{tabular}

Table 2: Key technologies used to improve the learning process.

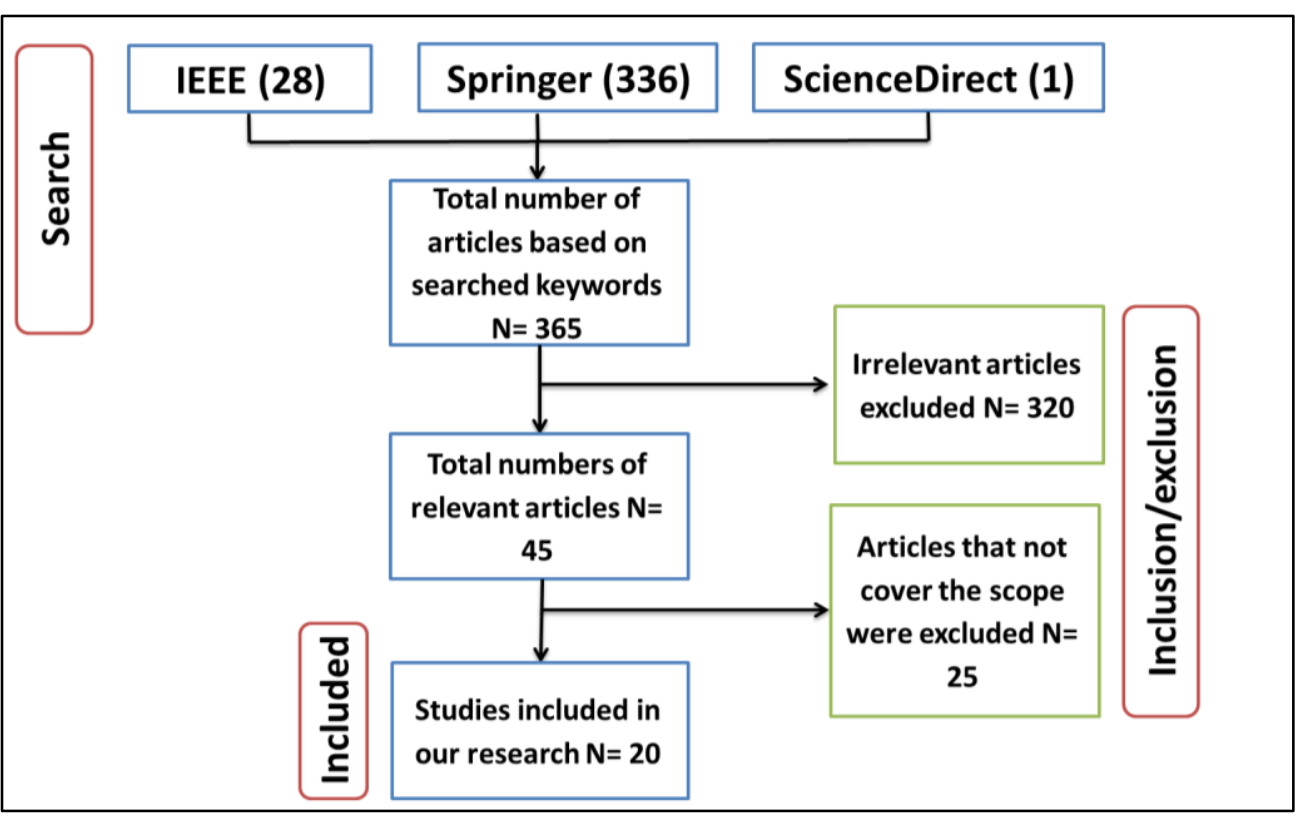

Figure 2: Articles from the most popular scientific databases.

selections by abstract and conclusion and finally based on the full-text reading. Results a total of 365 articles were found from the three scientific databases. After that, irrelevant articles were checked and excluded based on title and Abstract, has resulted in a left of 320 articles. The screening process continued during the full-text reading articles that resulting in 25 are excluded. Finally, 20 selected papers were used for this review paper as shown in figure 2.

\subsection{Classification by scientific databases}

Classification of articles by scientific databases was presented in Figure 2 IEEE introduces more than 50\% (12 out of 20 research papers or 60\%), Springer Link represents (7 out of 20 research papers, or 35\%), and ScienceDirect (1 out of 20 research, or $5 \%$ ) of the total number of articles. The retrieved articles reflecting the importance of using blockchain technology in the learning environment.

All articles represent the crucial rule of using semantic web blockchain technology to develop a new ecosystem of the learning environment.

\section{A proposed framework of learning process based on semantic blockchain (LSSB)}

The proposed framework integrates the semantic web with blockchain to enhance the effectiveness and efficiencies of the learning process. The semantic web is used to promote data interoperability by improving the representation capability of the learning process-based blockchain components by annotating them with semantically rich languages. The semantic web and blockchain are complementary technologies. Blockchain 


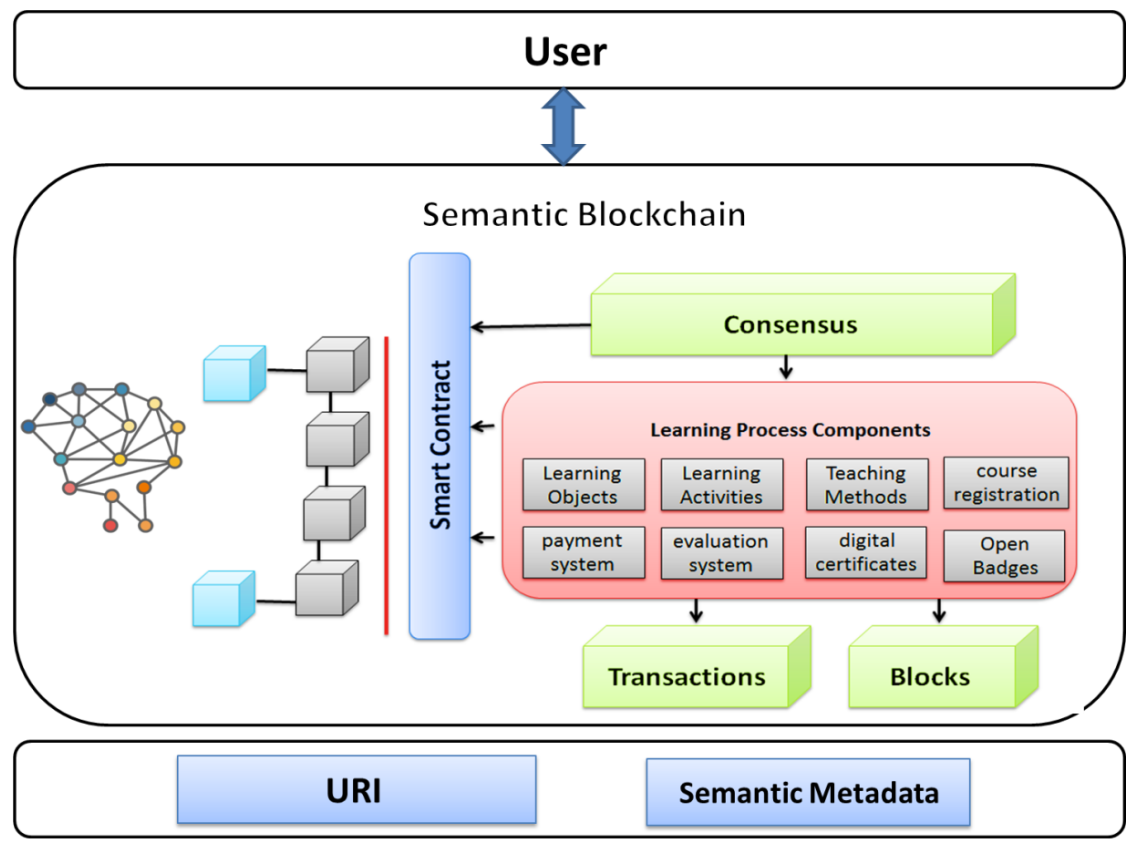

Figure 3: The proposed framework.

is being utilized to address some of the semantic web's open concerns, such as trust and identity management. The semantic web is used to improve the blockchain representation like annotations and data integration. These technologies are considered the backbone of the learning environments. The semantic blockchain introduces distributed, secure, transparent, real-time, immutable, and tamper-proof records of the learning process transactions. The proposed framework consists of three layers and is used to represent all components of the learning process as shown in figure 3 .

\subsection{Layer 1: Uniform Resource Identifier (URI)}

The semantic web employs URIs to represent resources and assign a unique identification to each one. It is a globally unique identifier that is used to consolidate all data associated with a specific resource, making it easier to combine data sources that were developed independently. While URI is very valuable, it has some inherent challenges such as centralization. Generating the URI depends on the centralized Domain Name System (DNS) that forms a single point of attack or failure. Blockchain provides a decentralized authority to control identifier creation and prevent the existence of a single point of attack or failure. URI identifies the knowledge representation of the educational domain-based blockchain, including concepts, sub-concepts, properties, and individuals.

\subsection{Layer 2: semantic blockchain}

It was difficult for participants to trace transactions on the blockchain, the formal representation of the learning process-based blockchain improves analysis capabilities and transparency. This layer introduces the semantic representations of whole components, relationships, and transactions of the learning process-based blockchain.
Ontology is used to introduce a shared and reused understanding of the concepts and sub-concepts of the learning process based on a blockchain [23-24]. It facilitates interlinking with other Linked data to perform formal reasoning and inferences. The knowledge related to the educational domain-based blockchain is represented by web ontology language (OWL). It contains participants, their roles, and the way they interact and communicate in the whole learning process transactions. These transactions include protecting learning objects, learning activities, teaching methods, course registration, payment system, evaluation system, and issue digital certificates or digital badges. This layer consists of learning process components, consensus techniques, transactions, blocks, and smart contracts.

\subsubsection{The learning process components}

Semantic blockchain plays an important role to store immutable and accurate records about the learning process components, which include the following:

1. Learner's records and profiles: educational institutions store learner data in a centralized database, which enables hackers to edit the grades and fake certifications. Blockchain technology introduces unique advantages containing decentralization, reliability, security, and data integrity. It enables educational institutions to store a real copy of the degree with a virtual copy.

2. Learning object, Learning Activities, and Teaching Methods: the semantic blockchain is used to protect learning objects, learning activities, and teaching methods acquired from professors or learners.

3. Evaluation System: (allows assessing the degree of achievement of the learning goal).

4. Certificates: The certificate is strong evidence for the learner that completes the course successfully. It is issued on paper includes all the details about the 
participants and educational institutions. It is then saved without being shared with others. The semantic blockchain is used to implement a new environment for these certificates, by issuing the certificates in digital format. All the identities of certification are stored in the semantic blockchain [16]. The semantic blockchain makes the authenticity of certifications is more easily verified. Once the professor stores a certificate in the semantic blockchain, the details become publicly available, immutable, permanent, and separated from the issuing establishment. It facilitates for employers to reference the online record to verify a potential learning achievement.

5. Payment System: the learning process with the blockchain is a secure and trustworthy place where educational institutions do not need a third party to manage the transfer of credits.

6. Digital Badges: A digital badge is defined as a powerful tool to validate the learners' skills, achievements, and knowledge because of completing the course and passing a quiz.

7. Publications: these are relevant to academic institution research.

All these data are stored within a chain of a sequence of blocks, where each new block is connected to the previous block to store data related to the learning process. For Example, A learner block contains his personal data like Name, Address, Email, and date of birth [8].

\subsubsection{Consensus technique}

First, we must identify the participants in the learning process. They include learners, educational institutions, professors, employees, and employers.

The process starts as soon as a learner participates in a course. After he completes the course, a new block is created, which contains the learners' results, the course information, and the time when the course was finished. The result could also be a certificate if the course was completed successfully. When a new block is created, all nodes in the network will receive this new block.

Consensus protocol (Proof-of-Work) enables participants in semantic, blockchain to agree to add a new block to the chain. It enables them to validate the correctness of the new block to confirm that it has not been modified and then add this block to their blockchain and the tampered blocks will be rejected.

\subsubsection{Smart Contracts}

A smart contract is used to manage the relationships between participants in the semantic blockchain network and determine the role of each participant. It is considered the main component in the semantic blockchain. It is a computer program, which consists of lines of code to digitally facilitate, verify, and enforce some type of agreement. It is executed independently and automatically when predetermined conditions and terms are met without an intermediary's involvement. A smart contract is used to secure learners' academic records, manage the process of registration, payments, and evaluations. It is used to validate whether a learner has finished the requirements for acquiring the required certificate. The smart contracts cannot be altered or controlled once it is deployed. This supports developing a trusted learning process [27]. All these components are represented using Web Ontology Language (OWL) as shown in the following section.

\section{Implementation}

Here we implement a small prototype of the proposed Framework (Learning Process Based on Semantic Blockchain (LSSB)) which is developed from scratch using protégé 5. All the required concepts and subconcepts, data properties, object properties, and the relations between concepts and sub-concepts and the properties of these relations related to the learning process-based blockchain domain are represented in figures 4 and 5.

The learning process-based semantic blockchain is created as mentioned in the following steps:

STEP-I: Figure 4 depicts all concepts and subconcepts connected to the learning process-based

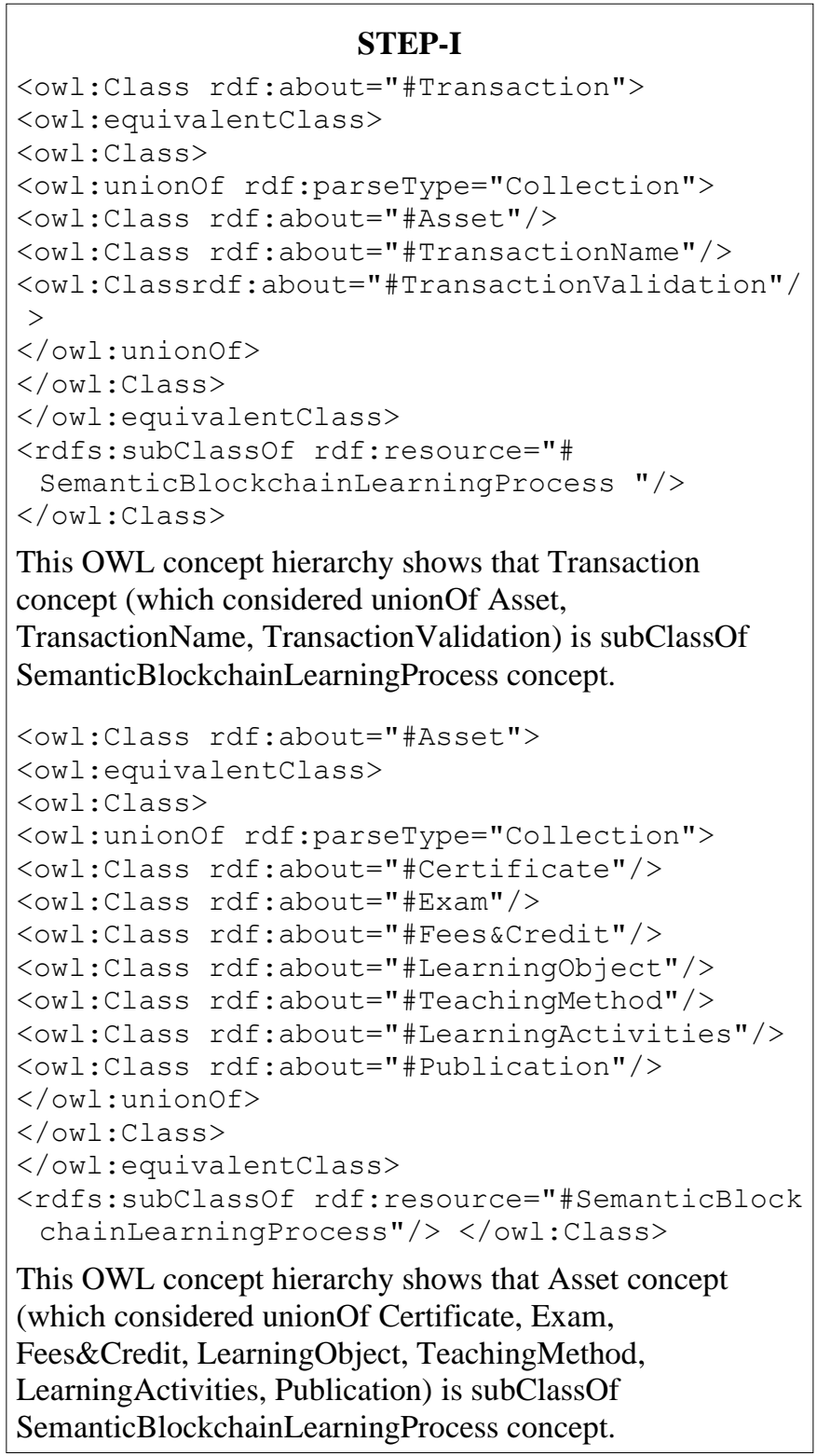




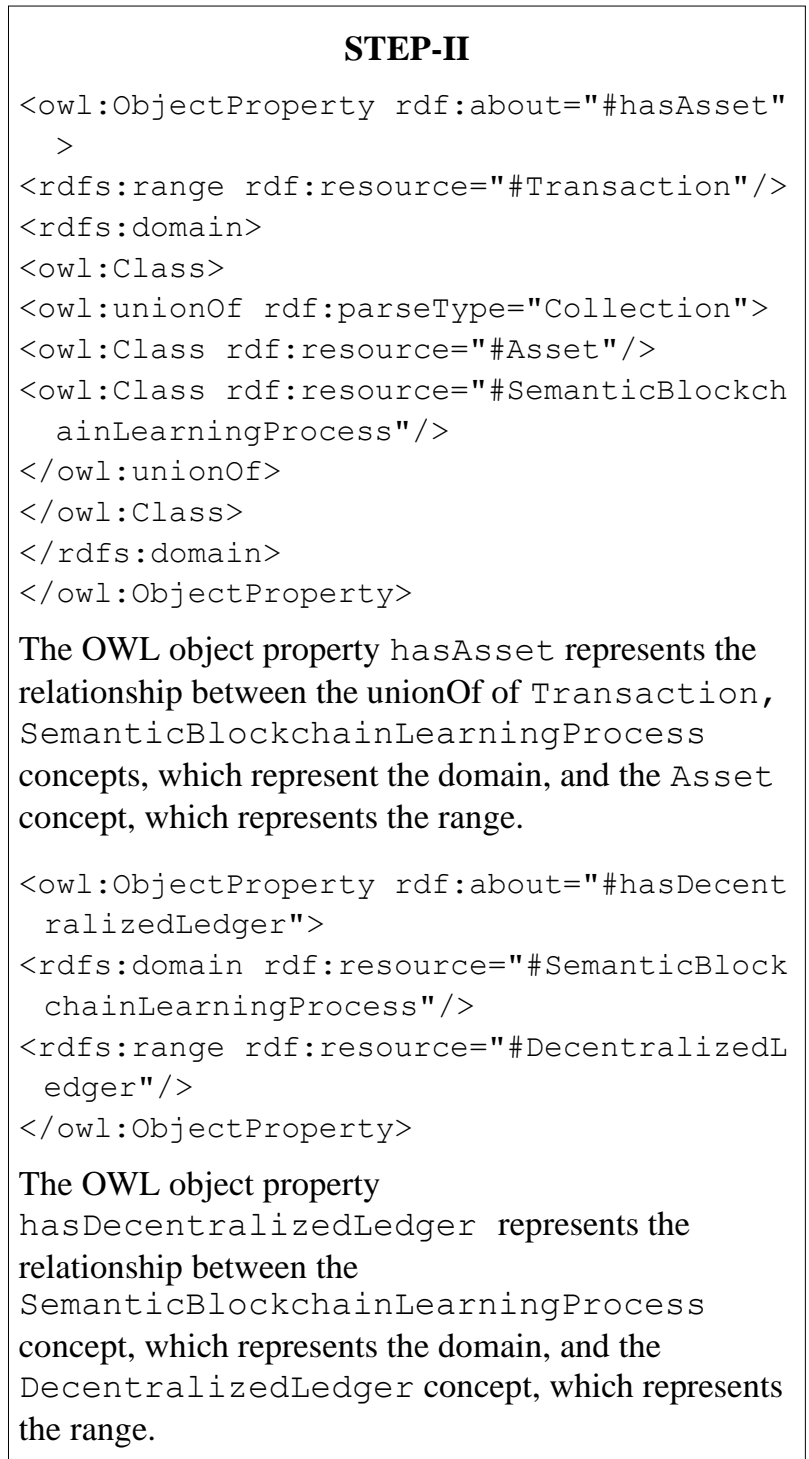

blockchain domain. All the concepts and sub-concepts are mainly focused on representing knowledge related to the whole components of the learning process. The OWL code below is an example of how all the concepts and subconcepts are represented using OWL language.

STEP-II: Represents object properties of the learning process based semantic blockchain. Describing the object properties is based on the relationship, which we want to add between concepts and sub-concepts of the learning process as shown in figure 5. As seen in the following example, these relationships are represented using domain and range in OWL:

STEP-III: is used to create data type properties of the ontology, the data type properties are used to define the relationship between an individual and a primitive data type. This relationship is represented using domain and range using OWL as shown in the following: The following OWL represents an excerpt from the Block data such as BlockID, Name, Hash, PreviousHash, TimeStamp, Transaction, and Validation and their data values. OWL-VIZ is used to visualize the learning process-based blockchain as shown in figure 6.

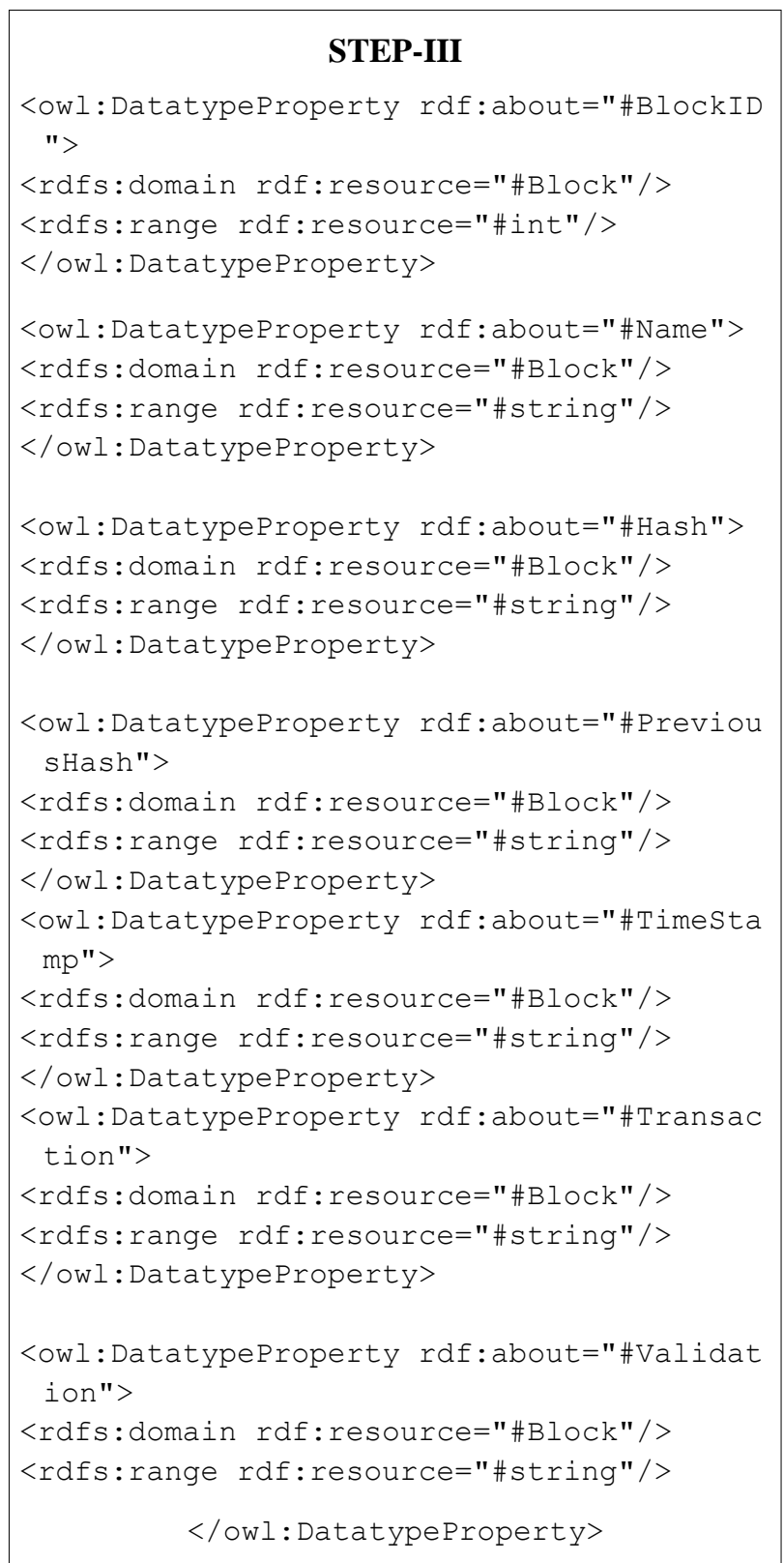

\section{Conclusion}

E-learning settings have evolved considerably, and there is a need to educate learners about the relevance of online teaching and learning, as well as connect learners and educators who were previously separated. The demand for digital transformation in educational institutions has risen to unprecedented levels in the time of COVID -19 . Literature review mentioned that many educational institutions improve their learning process by using IoT and blockchain ignored the integration of semantic web technology. This paper focused on representing the leading role of integrating the two technologies, which are considered crucial to assure an effective and efficient learning process. The proposed system makes use of blockchain and the semantic web to provide immutable distributed storage for tracing and tracking all transactions that comprise the learning process. The semantic web is used to promote information interoperability by 


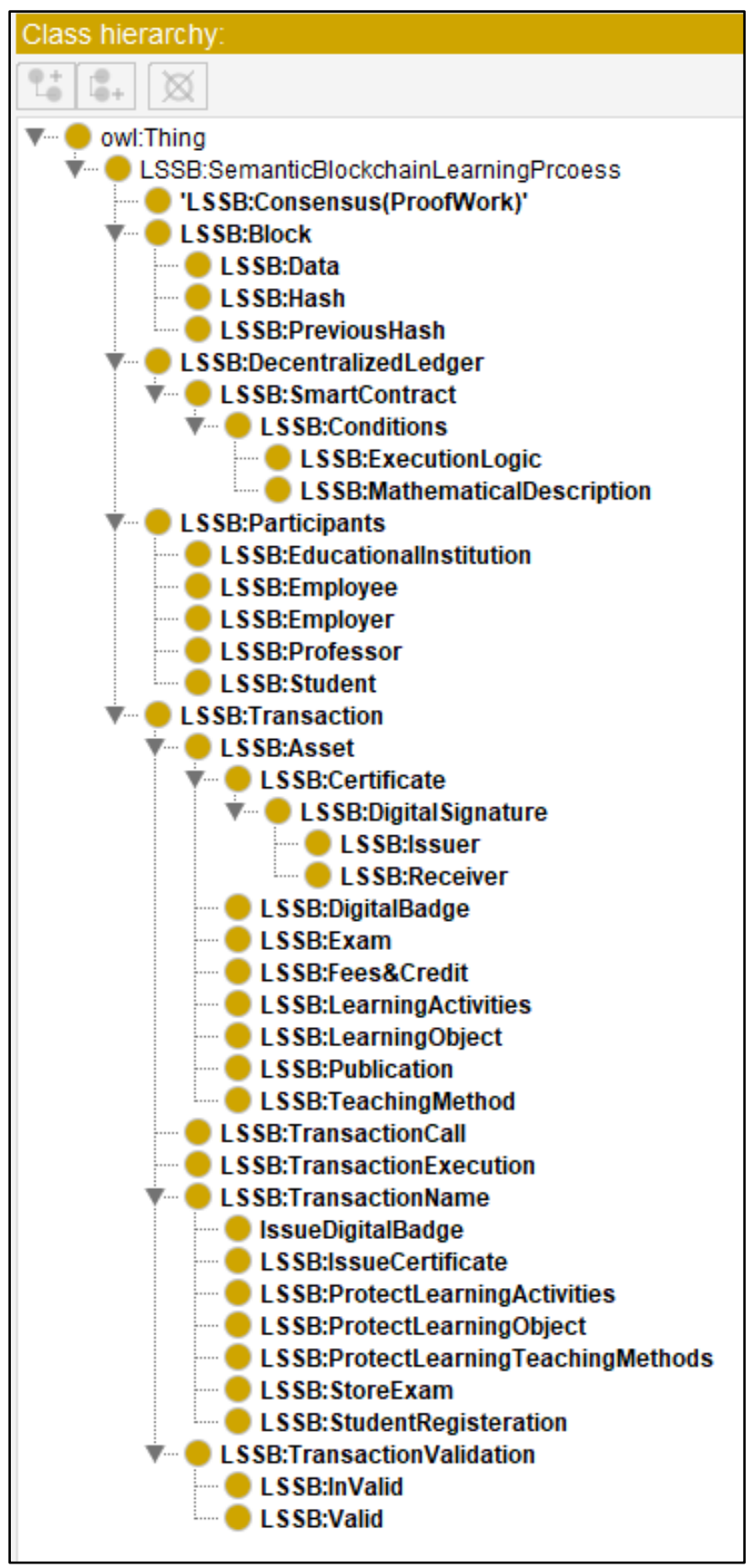

Figure 4: An excerpt of the learning process hierarchy based semantic blockchain.

improving the representation capability of the learning process and blockchain components by annotating them with semantically rich languages. Extra research will implement the full layers of the proposed framework and applying Semantic Web Rule Language (SWRL) to infer the new knowledge.

\section{Acknowledgment}

The author declares that she has no known competing financial interests or personal relationships that could have appeared to influence the work reported in this paper.

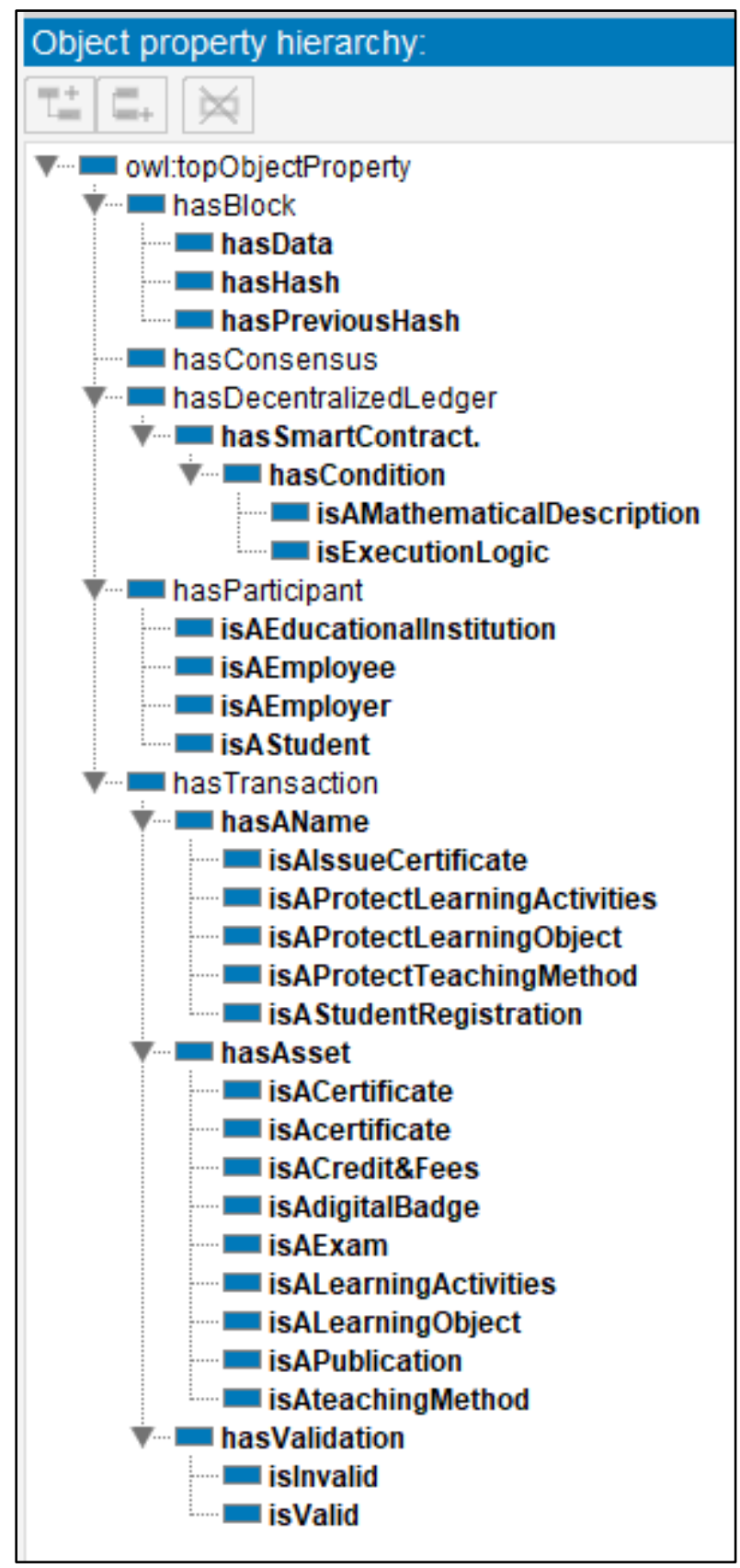

Figure 5: The objectt properties of learning process based semantic blockchain. 


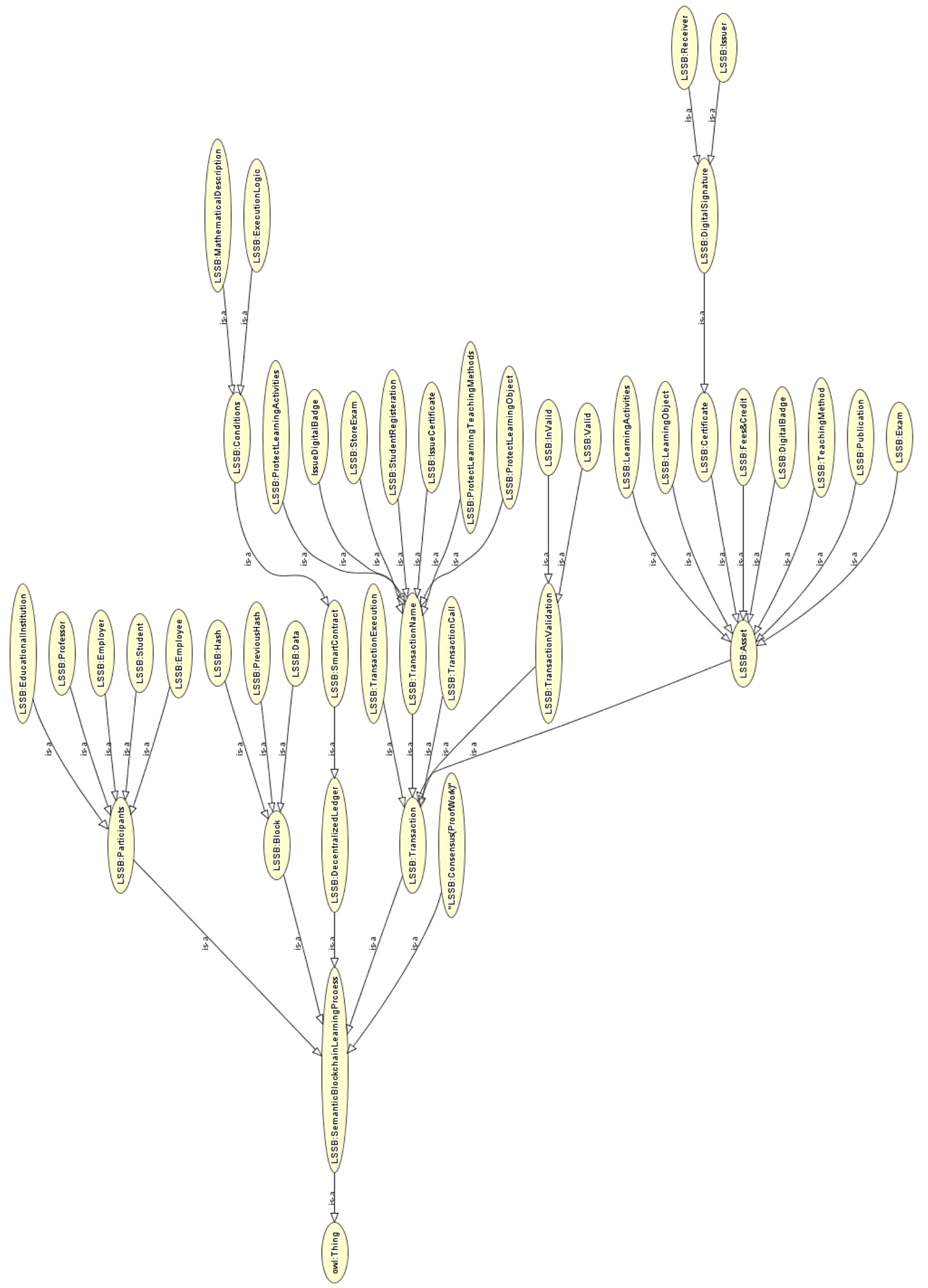

Figure 6: The visualization learning process is based on semantic blockchain. 


\section{References}

[1] Favale, T., Soro, F., Trevisan, M., Drago, I., \& Mellia, M. (2020). Campus Traffic and e-Learning during COVID-19 Pandemic. Computer Networks, 107290. https://doi.org/10.1016/j.comnet.2020.107290

[2] Chiodini, J. (2020). Online learning in the time of COVID-19. Travel Medicine and Infectious Disease. https://doi.org/10.1016/j.tmaid.2020.101669

[3] Widianto, A. A., Purwasih, J. H. G., \& Perguna, L. A. (2020). Promoting Social Cohesion: The Development of E-Learning Management System Materials through Life Based Learning for Sociology of Religion Course. International Journal of Emerging Technologies in Learning (iJET), 15(07), 162-170. https://doi.org/10.3991/ijet.v15i07.13339

[4] Tapscott, D. and A. Kaplan (2019). "Blockchain Revolution In Education And Lifelong Learning.". BLOCKCHAIN RESEARCH INSTITUTE - IBM INSTITUTE FOR BUSINESS VALUE.

[5] Zheng, Z., et al. (2018). "Blockchain challenges and opportunities: A survey." International Journal of Web and Grid Services 14(4): 352-375.

DOI: 10.1504/IJWGS.2018.10016848

[6] Rezgui, K., et al. (2018). "Towards a common and semantic representation of e-portfolios." Data Technologies and Applications 52(4): 520-538.

DOI 10.1108/DTA-01-2018-0008

[7] Hori, M., et al. (2018). Learning System based on Decentralized Learning Model using Blockchain and SNS. CSEDU (1). https://doi.org/10.5220/0006666901830190

[8] Mikroyannidis, A., et al. (2018). A Learner-Centred Approach for Lifelong Learning Powered by the Blockchain. EdMedia+ Innovate Learning, Association for the Advancement of Computing in Education (AACE).

[9] Sharples, M., \& Domingue, J. (2016, September). The blockchain and kudos: A distributed system for educational record, reputation and reward. In European conference on technology enhanced learning (pp. 490-496). Springer, Cham. DOI: 10.1007/978-3-319-45153-4_48

[10] Bdiwi, R., et al. (2017). Towards a new ubiquitous learning environment based on Blockchain technology. IEEE 17th International Conference on Advanced Learning Technologies (ICALT), IEEE. DOI: 10.1109/ICALT.2017.37

[11]Duan, B., et al. (2017). Education application of blockchain technology: Learning outcome and metadiploma. IEEE 23rd International Conference on Parallel and Distributed Systems (ICPADS), IEEE. DOI: 10.1109/ICPADS.2017.00114

[12] Turkanović, M., et al. (2018). "EduCTX: A blockchain-based higher education credit platform." IEEE access 6: 5112-5127.

DOI: 10.1109/ACCESS.2018.2789929

[13] Wu, B. and Y. Li (2018). Design of Evaluation System for Digital Education Operational Skill Competition Based on Blockchain. IEEE 15th
International Conference on e-Business Engineering (ICEBE), IEEE.

DOI: 10.1109/ICEBE.2018.00025

[14] Bdiwi, R., et al. (2019). Blockchain-Based Platform for Smart Learning Environments. International Conference on Business Information Systems, Springer.

https://doi.org/10.1007/978-3-030-20485-3_38

[15] Guo, J., et al. (2019). "Blockchain-enabled digital rights management for multimedia resources of online education." Multimedia Tools and Applications: 1-21. https://doi.org/10.1007/s11042-019-08059-1

[16] Hericko, M. A (2019). Preliminary Review of Blockchain-Based Solutions in Higher Education. Learning Technology for Education Challenges: 8th International Workshop, LTEC, Zamora, Spain, July 15-18, 2019, Proceedings, Springer. https://doi.org/10.1007/978-3-030-20798-4_11

[17] Yumna, H., et al. (2019). Use of Blockchain in Education: A Systematic Literature Review. Asian Conference on Intelligent Information and Database Systems, Springer. https://doi.org/10.1007/978-3-030-14802-7_17

[18] Al Harthy, K., et al. (2019). The upcoming Blockchain adoption in Higher-education: requirements and process. 4th MEC International Conference on Big Data and Smart City (ICBDSC), IEEE. DOI: 10.1109/ICBDSC.2019.8645599

[19] Bdiwi, R., et al. (2018). A Blockchain Based Decentralized Platform for Ubiquitous Learning Environment. IEEE 18th International Conference on Advanced Learning Technologies (ICALT), IEEE. DOI: 10.1109/ICALT.2018.00028

[20]Gong, X., et al. (2018). Parallel-EducationBlockchain Driven Smart Education: Challenges and Issues. Chinese Automation Congress (CAC), IEEE. doi: 10.1109/CAC.2018.8623198

[21] Liu, Q., et al. (2018). Education-Industry Cooperative System Based on Blockchain. 1st IEEE International Conference on Hot Information-Centric Networking (HotICN), IEEE.

DOI 10.1108/ITSE-07-2020-0102

[22] Srivastava, A., et al. (2018). A Distributed Credit Transfer Educational Framework based on Blockchain. Second International Conference on Advances in Computing, Control and Communication Technology (IAC3T), IEEE. doi: 10.1109/IAC3T.2018.8674023.

[23] Mikroyannidis, A., et al. (2018). Smart Blockchain Badges for Data Science Education. IEEE Frontiers in Education Conference (FIE), IEEE. doi: 10.1109/FIE.2018.8659012.

[24] Juričić, V., et al. (2019). Creating learner's profile using blockchain technology. 2019 42nd International Convention on Information and Communication Technology, Electronics and Microelectronics (MIPRO), IEEE. https://doi.org/10.1186/s41039-019-0097-0 
[25] Daoudi, N., Nabigh, M., Hilal, I., Rahimi, H., \& Atiki, F. Z. (2020). Organizational Intelligence: Ontological Modeling of Skills. International Journal of Advanced Corporate Learning (iJAC), 13(1), 4-18. DOI: 10.3991/ijac.v13i1.10589

[26] Assami, S., Daoudi, N., \& Ajhoun, R. (2020). A Semantic Recommendation System for Learning Personalization in Massive Open Online Courses. International Journal of Recent Contributions from Engineering, Science \& IT (iJES), 8(1), 71-80.
DOI:10.3991/ijes.v8i1.14229

[27] Palma, L. M., et al. (2019). "Blockchain and smart contracts for higher education registry in Brazil." International Journal of Network Management 29(3): e2061.

https://doi.org/10.1002/nem.2061 